\title{
Crecimiento y supervivencia de Cryphiops caementarius en cocultivo con Oreochromis niloticus a diferentes densidades
}

\author{
Anghela Mogollón-Calderón ${ }^{凶(\mathbb{D}}$ Biol Acui; Walter Reyes-Avalos ${ }^{1 *} \bowtie(\mathbb{C}$ Ph.D.
}

\begin{abstract}
${ }^{1}$ Universidad Nacional del Santa. Facultad de Ciencias. Escuela Profesional de Biología en Acuicultura. Departamento Académico de Biología, Microbiología y Biotecnología. Laboratorio de Acuicultura Ornamental. Avenida Universitaria s/n Urbanización Bellamar, Nuevo Chimbote, Ancash, Perú.
\end{abstract}

*Correspondencia: wreyes@uns.edu.pe

Recibido: Marzo 2020; Aceptado: Noviembre 2020; Publicado: Marzo 2021.

\section{RESUMEN}

Objetivo. Evaluar el crecimiento y la supervivencia de Cryphiops caementarius en cocultivo con Oreochromis niloticus a diferentes densidades. Materiales y métodos. Se utilizaron camarones machos (5.86 cm y $7.65 \mathrm{~g}$ ) y alevines revertidos de tilapia (5.65 cm y $2.61 \mathrm{~g}$ ). Se emplearon nueve acuarios ( $55 \mathrm{~L}$ ). En cada acuario se instalaron seis recipientes donde se sembró un camarón por recipiente (32 camarones $/ \mathrm{m}^{2}$ ) y en el agua restante se sembraron tilapias a 100, 200 y 300 alevines $/ \mathrm{m}^{3}$. Se empleó alimento balanceado. La ración diaria para camarones fue del $6 \%$ y para tilapia fue del $5 \%$ de la biomasa total. El experimento duró 90 días. Resultados. En el camarón, la longitud $(6.46 \mathrm{~cm})$, peso $(9.37 \mathrm{~g})$, las ganancias porcentuales en longitud $(10.01 \%$ a $10.45 \%)$ peso $(19.24 \%$ a $25.41 \%)$, y la supervivencia $(88.89 \%$ a $94.44 \%)$ fueron similares $(p<0.05)$ entre tratamientos. El efecto del síndrome de muerte por muda es discutido. En tilapia, la longitud ( 9.25 $\mathrm{cm})$, peso $(12.90 \mathrm{~g})$, tasa de crecimiento absoluto $(0.040 \mathrm{~cm} /$ día; $0.114 \mathrm{~g} /$ día $)$, tasa de crecimiento específica $(0.55 \%$ longitud/día; $1.759 \%$ peso/día) y la ganancia porcentual $(64.21 \% ; 389.48 \%)$ fueron mayores $(p<0.05)$ a 100 y 200 alevines $/ \mathrm{m}^{3}$. La supervivencia de tilapia fue similar $(86.11 \%)$ entre tratamientos. Conclusiones. El crecimiento y la supervivencia del camarón fueron afectados por el síndrome de muerte por muda, más no por la presencia de tilapia en el sistema. En cambio, mayor crecimiento de tilapia se obtuvo con 100 alevines $/ \mathrm{m}^{3}$ y la supervivencia fue similar entre tratamientos.

Palabras clave: Cultivo intensivo; biomasa; policultivo; camarón; tilapia. (Fuente: CAB).

\section{ABSTRACT}

Objective. To evaluate the growth and survival of Cryphiops caementarius in coculture with Oreochromis niloticus at different densities. Materials and methods. Male prawns $(5.86 \mathrm{~cm}$ and $7.65 \mathrm{~g})$ and reverse tilapia fingerlings $(5.65 \mathrm{~cm}$ and $2.61 \mathrm{~g})$ were used. Nine aquariums $(55 \mathrm{~L})$ were used. Six containers were installed in each aquarium, where one prawn was stocked per container (32 prawn $/ \mathrm{m}^{2}$ ), and in the remaining water, tilapia was stocked at densities of 100, 200 and 300 fish $/ \mathrm{m}^{3}$. Balanced feed was used. The daily ration for prawns was $6 \%$ and for tilapia, it was $5 \%$ of the total 
biomass. The experiment lasted 90 days. Results. In prawns, the length $(6.46 \mathrm{~cm})$, weight $(9.37 \mathrm{~g})$, percentage gains in length $(10.01 \%$ at $10.45 \%)$ weight $(19.24 \%$ a $25.41 \%)$, and survival $(88.89 \%$ to $94.44 \%)$ were similar $(p<0.05)$ between treatments. The effect of molting death syndrome is discussed. In tilapia, the length $(9.25 \mathrm{~cm})$, weight $(12.90 \mathrm{~g})$, absolute growth rate $(0.040 \mathrm{~cm} /$ day; $0.114 \mathrm{~g} /$ day $)$, specific growth rate $(0.55 \%$ length/day; $1.759 \%$ weight/day) and percentage gain $(64.21 \% ; 389.48 \%)$ were greater $(p<0.05)$ at 100 and 200 fish/m $\mathrm{m}^{3}$. Tilapia survival was similar $(86.11 \%)$ between treatments. Conclusions. Prawn growth and survival were affected by molt death syndrome but not by the presence of tilapia in the system. In contrast, greater growth of tilapia was obtained with 100 fish $/ \mathrm{m}^{3}$, although survival was similar between treatments.

Keywords: Intensive culture; biomass; polyculture; prawn; tilapia. (Source: $C A B$ ).

\section{INTRODUCCIÓN}

El cocultivo es similar al policultivo por la combinación de especies, pero difieren en la manera como se separan dentro del ambiente acuático. En el policultivo los organismos por ser de hábitos alimenticios y de distribución espacial diferentes, maximizan la producción, donde una especie es la principal y la otra no, pero se consideran en el rendimiento (1). En el cocultivo, dos o más especies ocupan el mismo cuerpo de agua, pero hay separación física entre ellas, por ejemplo, una especie cultivada en jaulas dentro de un estanque donde se cultiva otra especie (2), y en este caso las especies son importantes en el manejo y en la producción.

En el cocultivo de Homarus gammarus en compartimientos dentro de tanques con Idotea emarginata, este último utiliza los desechos generados en el sistema (3). En el cocultivo de Clarias batrachus en jaulas dentro de estanques con Macrobrachium rosenbergii, se obtuvo mayor crecimiento y productividad (4). Así mismo, en el cocultivo de Oreochromis niloticus en jaulas dentro de estanques con $M$. rosenbergii, ambas especies muestran mayor crecimiento y rendimiento al no haber interacción física (5). Sin embargo, no se ha investigado el cocultivo de Cryphiops caementarius en compartimentos individuales dentro de acuarios con diferentes densidades de $O$. niloticus.

La tilapia 0 . niloticus es omnívora, pero se alimenta de microalgas que se encuentran en la columna del agua y en el substrato (6). Además, esta especie de tilapia es resistente y de rápido crecimiento en diferentes sistemas de manejo, pero con adecuadas densidades de siembra. La tilapia a 50 peces $/ \mathrm{m}^{3}$ muestra mayor peso final y tasa de crecimiento específico que a mayores densidades (7) y entre 100 y 450 peces $/ \mathrm{m}^{3}$ no se afecta el crecimiento y el rendimiento
$(8,9,10)$. En 0 . mossambicus el incremento de 429 a 1716 peces $/ \mathrm{m}^{3}$ ocasiona disminución del crecimiento por la jerarquía social que provoca que los peces pequeños no se alimenten debido a la dominancia de los más grandes (11).

El camarón $C$. caementarius habita los ríos desde Lambayeque en Perú hasta Valparaíso en Chile (12), pero alta densidad poblacional se encuentra en los ríos de Arequipa, Perú, donde la temperatura del agua varía entre 18.4 a $26.8^{\circ} \mathrm{C}$ (13). Esta especie de camarón tiene importancia económica y comercial, pues actualmente ingresaron $997 \mathrm{t}$ al mercado de Lima, Perú (14). El cultivo del camarón no se ha establecido por la agresividad de los ejemplares machos que conduce a la interacción y al canibalismo. Sin embargo, una alternativa para mejorar el crecimiento, la supervivencia y el rendimiento productivo es mediante el uso de recipientes individuales donde el cultivo es en alta densidad (32 camarones $/ \mathrm{m}^{2}$ ) (15). En el cultivo de crustáceos agresivos en compartimentos individuales, no hay canibalismo, el crecimiento es mayor y se mantiene alta supervivencia aun en densidades muy altas (>142 camarones/ $\mathrm{m}^{2}$ ) como en Cherax quadricarinatus (16) y $\mathrm{H}$. gammarus (17).

En consecuencia, de acuerdo con los avances en el cultivo del crustáceo de clima templado (C. caementarius) y del pez tropical ( $O$. niloticus), el objetivo fue evaluar el crecimiento y supervivencia de $C$. caementarius en cocultivo con $O$. niloticus a diferentes densidades.

\section{MATERIALES Y MÉTODOS}

Organismos. Los camarones machos se colectaron del río Pativilca (10³9'53.6" S - 77040'23.2" W) cerca del centro poblado Huayto (Lima, Perú) y para el transporte, cada 
camarón se introdujo en un vaso de plástico con orificios $(250 \mathrm{~mL})$ y fueron acondicionados dentro de recipientes con agua del mismo río (45 L) y aireación continua (15). La densidad de transporte fue de 50 camarones por recipiente. El transporte terrestre duró $5 \mathrm{~h}$ y no hubo mortalidad. Los camarones se aclimataron por una semana en el mismo sistema de transporte y se alimentaron con balanceado que fue formulado previamente (30\% proteína cruda, $5 \%$ de grasa total, $10 \%$ de cenizas y $5 \%$ de fibra) (15). La especie de $C$. caementarius se reconoció con clave taxonómica (18) y el sexo del macho se verificó por la presencia de gonóporos en los coxopoditos del quinto par de periópodos (19).

Los alevines machos de 0 . niloticus, revertidos sexualmente, de 20 días de edad, procedieron de la Estación Pesquera Ahuashiyacu (6³0'55.83" S - 76¹9'50.65" W) (Tarapoto, Perú). Los alevines se transportaron en bolsas de polietileno con $10 \mathrm{~L}$ de agua ( 25 alevines/L) y oxígeno puro a presión. Las bolsas con peces se acondicionaron dentro de baldes de plástico de $20 \mathrm{~L}$. El transporte vía terrestre duró $30 \mathrm{~h}$ y hubo $5 \%$ de mortalidad. Los alevines se aclimataron, durante una semana, en tanques de fibra de vidrio ( $240 \mathrm{~L})$ a 200 alevines/ $\mathrm{m}^{3}$ y se alimentaron a saciedad con balanceado.

Cocultivo. El sistema de cocultivo empleado (15) consistió de nueve acuarios de vidrio ( 0.60 $\mathrm{m}$ de largo, $0.31 \mathrm{~m}$ de ancho, $0.35 \mathrm{~m}$ de alto, de $0.186 \mathrm{~m}^{2}$ y $55 \mathrm{~L}$ ), cada uno con sistema de recirculación de agua tipo air-water-lift (1.5 L/ min) y con filtro biológico percolador $(2.5 \mathrm{~L})$ cuyo lecho filtrante fue conchuela triturada y grava, en iguales proporciones. Dentro de cada acuario se instalaron seis recipientes de plástico $(19 \mathrm{~cm}$ de diámetro, $8 \mathrm{~cm}$ de altura y $284 \mathrm{~cm}^{2}$ ) que se dispusieron en dos grupos de tres niveles. Los recipientes tuvieron orificios ( $3 \mathrm{~cm}$ de largo por $0.5 \mathrm{~cm}$ de ancho) en los laterales para permitir el flujo de agua, además se adhirió un tubo (PVC de $1 / 2 " \varnothing)$ que sobresalió el nivel del agua y por donde se introdujeron los gránulos de alimento (Figura 1). Los seis recipientes instalados ocuparon $15 \mathrm{~L}$ y el volumen de agua restante del acuario fue de $40 \mathrm{~L}$. El agua potable fue aireada durante $72 \mathrm{~h}$ para eliminar el cloro.

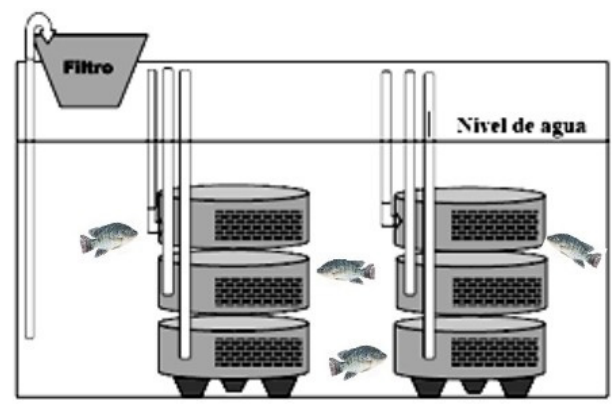

Figura 1. Sistema del cocultivo de $C$. caementarius con $O$. niloticus en acuario.

Siembra. En cada recipiente de cultivo se sembró un camarón, que equivalió a seis camarones por acuario (32 camarones $/ \mathrm{m}^{2}$ ). Se emplearon 54 camarones $(5.86 \pm 0.12 \mathrm{~cm}$ y $7.65 \pm 0.26 \mathrm{~g})$ con los apéndices cefalotorácicos completos sin signos de laceraciones en el cuerpo y apéndices. Además, en cada acuario se sembraron 4, 8 y 12 alevines de tilapia, que equivalieron a 100 , 200 y 300 alevines $/ \mathrm{m}^{3}$, respectivamente. Se emplearon 72 alevines de apariencia saludable $(5.65 \pm 0.03 \mathrm{~cm}$ y $2.61 \pm 0.08 \mathrm{~g})$.

Alimento. Los camarones y las tilapias se alimentaron con el mismo balanceado que se preparó con la formulación para camarón (15) suplementado con 3\% de Saccharomyces cerevisiae (20) y el cual tuvo $30 \%$ de proteína cruda, $8.1 \%$ de lípidos totales y $4.6 \%$ de fibra, con $2600 \mathrm{kcal} / \mathrm{g}$. La ración diaria (08:00 y 18:00 h) para camarón fue del $6 \%$ del peso húmedo y para tilapia del $5 \%$. El alimento para camarones se distribuyó por los tubos alimentadores de cada recipiente, y para tilapia por la superficie del agua del acuario.

Parámetros zootécnicos. Los muestreos de camarón y tilapia se realizaron cada 30 días, durante 90 días. El peso total se determinó en balanza digital (Adam AQT600, $\pm 0.1 \mathrm{~g}$ ). La longitud total del camarón (desde escotadura post orbital hasta el extremo posterior del telson) y de tilapia se midieron con regla graduada $( \pm 0.1 \mathrm{~cm})$. Los parámetros zootécnicos se determinaron con las siguientes fórmulas:

Ganancia porcentual $(\%)=\left[\left(\mathrm{X}_{2}-\mathrm{X}_{1}\right) / \mathrm{X}_{1}\right] * 100$

Tasa de crecimiento absoluto $=\left(\mathrm{X}_{2}-\mathrm{X}_{1}\right) / \mathrm{t}_{2}-\mathrm{t}_{1}$

Tasa de crecimiento específico (\%/día) $=\left[\ln X_{2}\right.$ $\left.\left.-\ln \mathrm{X}_{1}\right) / \mathrm{t}_{2}-\mathrm{t}_{1}\right] * 100$ 
Supervivencia $(\%)=\left(\mathrm{N}_{\mathrm{i}} * 100\right) / \mathrm{N}_{\mathrm{o}}$

Rendimiento $=\left(X_{2} * D_{2}\right) / 1000$

donde $X_{1}$ y $X_{2}$ son la longitud o el peso húmedo, inicial y final; $t_{1}$ y $t_{2}$ son la duración en días; In $X_{1} y$ In $X_{2}$ son el logaritmo natural de la longitud o el peso inicial y final; $\mathrm{N}_{\mathrm{o}}$ y $\mathrm{N}_{\mathrm{i}}$ son el número inicial y final de organismos sembrados, respectivamente; y $\mathrm{D}_{2}$ es la densidad final.

Calidad del agua. Los desechos sólidos acumulados en los acuarios se extrajeron semanalmente. La calidad del agua se monitoreó cada 15 días y se determinó oxígeno disuelto y temperatura del agua con oxímetro digital (Hatch $\left.\mathrm{LDO}, \pm 0.01 \mathrm{mg} / \mathrm{L}, \pm 0.01^{\circ} \mathrm{C}\right)$, y la dureza total, amonio total y nitritos con test colorimétrico Nutrafin $( \pm 0.1 \mathrm{mg} / \mathrm{L})$.

Análisis estadístico. Los datos se analizaron con la prueba de Shapiro-Wilk $(p<0.05)$ y todos cumplieron el supuesto de distribución normal. Para conocer si existieron diferencias entre las medias de los tratamientos se realizó análisis de varianza (ANOVA) de una vía y con la prueba de comparación múltiple de Tukey se determinó cuales fueron significativamente diferentes $(p<0.05)$. El procesamiento estadístico se realizó con el software SPSS versión 23 para Windows.

Aspectos éticos. En el procedimiento del experimento con animales vivos, se tuvo en cuenta la Ley peruana vigente (Ley 27265, Ley de Protección a los Animales Domésticos y a los Animales Silvestres Mantenidos en Cautiverio).

\section{RESULTADOS}

Cultivo de camarón. La longitud final promedio $(6.46 \pm 0.17 \mathrm{~cm} ; \mathrm{F}=0.126 ; \mathrm{p}=0.884)$ y el peso final promedio $(9.37 \pm 0.69 \mathrm{~g} ; \mathrm{F}=0.172$; $\mathrm{p}=0.846$ ) del camarón de todos los tratamientos fueron significativamente similares; de igual manera, sucedió con la tasa de crecimiento específica, la tasa de crecimiento absoluto y la ganancia porcentual. Sin embargo, las mayores ganancias porcentuales en longitud se obtuvieron en el cocultivo con tilapia a 100 y 200 alevines $/ \mathrm{m}^{3}$ (10.29 $4.02 \%$ y $10.45 \pm 3.00 \%$, respectivamente), y las ganancias porcentuales en peso con 200 y 300 alevines $/ \mathrm{m}^{3}(25.41 \pm 3.02 \%$ y $23.29 \pm 9.41 \%$, respectivamente). Similares tendencias se obtuvieron con la tasa de crecimiento específica y la tasa de crecimiento absoluto en longitud y en peso (Tabla 1 ). La supervivencia del camarón fue similar (92.59 $\pm 8.79 \% ; F=0.333$; $\mathrm{p}=0.729$ ) entre tratamientos, pero mayor supervivencia $(94.44 \pm 9.62 \%)$ se obtuvo en aquellos cocultivados a 100 y 200 alevines $/ \mathrm{m}^{3}$, y la menor supervivencia $(88.89 \pm 9.62 \%)$ con 300 alevines $/ \mathrm{m}^{3}$ (Tabla 1 ).

Tabla 1. Parámetros zootécnicos (media \pm desviación estándar) de $C$. caementarius en cocultivo con 0 . niloticus a diferentes densidades, durante 90 días.

\begin{tabular}{|c|c|c|c|}
\hline \multirow{2}{*}{ Parámetros } & \multicolumn{3}{|c|}{ Densidad de tilapia (alevines $/ \mathrm{m}^{3}$ ) } \\
\hline & 100 & 200 & 300 \\
\hline Longitud inicial (cm) & $\begin{array}{c}5.88 \\
\pm 0.10^{a}\end{array}$ & $\begin{array}{c}5.86 \\
\pm 0.16^{a}\end{array}$ & $\begin{array}{c}5.83 \\
\pm 0.14^{\mathrm{a}}\end{array}$ \\
\hline Longitud final (cm) & $\begin{array}{c}6.49 \\
\pm 0.30^{a}\end{array}$ & $\begin{array}{l}6.47 \\
\pm 0.11^{a}\end{array}$ & $\begin{array}{c}6.41 \\
\pm 0.09 a\end{array}$ \\
\hline TCE (\% longitud/día) & $\begin{array}{c}0.108 \\
\pm 0.041 \text { a }\end{array}$ & $\begin{array}{c}0.110 \\
\pm 0.030 \text { a }\end{array}$ & $\begin{array}{c}0.105 \\
\pm 0.043^{a}\end{array}$ \\
\hline TCA (cm/día) & $\begin{array}{c}0.007 \\
\pm 0.003^{a}\end{array}$ & $\begin{array}{c}0.007 \\
\pm 0.002 \text { a }\end{array}$ & $\begin{array}{c}0.006 \\
\pm 0.003^{a}\end{array}$ \\
\hline GP (\%) & $\begin{array}{c}10.29 \\
\pm 4.02^{\mathrm{a}}\end{array}$ & $\begin{array}{l}10.45 \\
\pm 3.00^{\mathrm{a}}\end{array}$ & $\begin{array}{l}10.01 \\
\pm 4.18^{\mathrm{a}}\end{array}$ \\
\hline Peso inicial (g) & $\begin{array}{c}7.87 \\
\pm 0.16^{a}\end{array}$ & $\begin{array}{l}7.62 \\
\pm 0.25^{a}\end{array}$ & $\begin{array}{c}7.46 \\
\pm 0.25^{a}\end{array}$ \\
\hline Peso final $(\mathrm{g})$ & $\begin{array}{c}9.37 \\
\pm 1.17^{a}\end{array}$ & $\begin{array}{l}9.56 \\
\pm 0.51^{\mathrm{a}}\end{array}$ & $\begin{array}{c}9.19 \\
\pm 0.44^{a}\end{array}$ \\
\hline TCE (\% peso/día) & $\begin{array}{c}0.189 \\
\pm 0.145 \text { a }\end{array}$ & $\begin{array}{r}0.251 \\
\pm 0.027 \text { a }\end{array}$ & $\begin{array}{c}0.230 \\
\pm 0.086 \text { a }\end{array}$ \\
\hline TCA (g/día) & $\begin{array}{c}0.016 \\
\pm 0.014^{\mathrm{a}}\end{array}$ & $\begin{array}{c}0.021 \\
\pm 0.003^{a}\end{array}$ & $\begin{array}{c}0.019 \\
\pm 0.007 a\end{array}$ \\
\hline GP (\%) & $\begin{array}{c}19.24 \\
\pm 15.82^{\mathrm{a}}\end{array}$ & $\begin{array}{l}25.41 \\
\pm 3.02^{a}\end{array}$ & $\begin{array}{l}23.29 \\
\pm 9.41^{\mathrm{a}}\end{array}$ \\
\hline Rendimiento $\left(\mathrm{kg} / \mathrm{m}^{2}\right)$ & $\begin{array}{c}0.302 \\
\pm 0.038^{a}\end{array}$ & $\begin{array}{c}0.308 \\
\pm 0.017 a\end{array}$ & $\begin{array}{c}0.263 \\
\pm 0.015^{a}\end{array}$ \\
\hline Supervivencia (\%) & $\begin{array}{c}94.44 \\
\pm 9.62^{\mathrm{a}}\end{array}$ & $\begin{array}{c}94.44 \\
\pm 9.62^{\mathrm{a}}\end{array}$ & $\begin{array}{c}88.89 \\
\pm 9.62^{a} \\
\end{array}$ \\
\hline
\end{tabular}

TCE: Tasa de crecimiento específica. TCA: Tasa de crecimiento absoluto. GP: Ganancia porcentual. Datos con diferentes letras en superíndice en una misma fila indica diferencia significativa $(p<0.05)$.

Las muertes de camarones se produjeron por ecdisis incompleta, porque la exuvia quedó atrapada en los periópodos o en los quelípodos. Sin embargo, algunos camarones cuya exuvia quedó atrapada en el quelípodo mayor, sobrevivieron porque realizaron autotomía de dicho quelípodo. Además, se observó que los camarones sacaban frecuentemente los quelípodos por los orificios del recipiente de cultivo con el cual intentaban capturar a las tilapias; pero, no hubo muertes de tilapias por este comportamiento del camarón. El rendimiento del camarón fue similar $\left(0.291 \pm 0.031 \mathrm{~kg} / \mathrm{m}^{2} ; \mathrm{F}=\right.$ $2.918 ; p=0.130$ ) entre tratamientos, pero mayores rendimientos fueron obtenidos en los cocultivados con tilapia a 100 y 200 alevines $/ \mathrm{m}^{3}(0.302 \pm 0.038$ y $0.308 \pm 0.017 \mathrm{~kg} / \mathrm{m}^{2}$, respectivamente), y el menor rendimiento $\left(0.263 \pm 0.015 \mathrm{~kg} / \mathrm{m}^{2}\right)$ se obtuvo en aquellos con 300 alevines $/ \mathrm{m}^{3}$ (Tabla 1 ). 
Cultivo de tilapia. El crecimiento en peso y longitud de tilapia varió inversamente proporcional con la densidad de siembra (Tabla 2). En tilapia cultivada a 100 alevines/ $\mathrm{m}^{3}$, los parámetros de crecimiento en longitud fueron significativamente mayores $(F=12.207$; $\mathrm{p}=0.008$ ) que aquellos a 200 y 300 alevines $/ \mathrm{m}^{3}$; de igual manera sucedió solo con el peso final $(12.90 \pm 1.13 \mathrm{~g})$ y la tasa de crecimiento absoluto en peso ( $0.114 \pm 0.013 \mathrm{~g} /$ día $)$. En cambio, la tasa de crecimiento específica (1.759 $\pm 0.133 \% /$ día) y la ganancia porcentual (389.48 $\pm 56.52 \%)$ en peso de aquellos cultivados a 100 alevines/ $\mathrm{m}^{3}$, fueron similares hasta con 200 alevines/ $\mathrm{m}^{3}(1.538 \pm 0.109 \% /$ día y $300.53 \pm 40.12 \%$ / día), pero diferentes con los de 300 alevines/ $\mathrm{m}^{3}$ (Tabla 2).

Tabla 2. Parámetros zootécnicos (media \pm desviación estándar) de $O$. niloticus a diferentes densidades en cocultivo con C. caementarius durante 90 días.

\begin{tabular}{cccc}
\hline \multirow{2}{*}{ Parámetros } & \multicolumn{3}{c}{ Densidad de tilapia (alevines $/ \mathbf{m}^{\mathbf{3}}$ ) } \\
\cline { 2 - 4 } & $\mathbf{1 0 0}$ & $\mathbf{2 0 0}$ & $\mathbf{3 0 0}$ \\
\hline Longitud inicial (cm) & 5.64 & 5.65 & 5.65 \\
& $\pm 0.04^{\mathrm{a}}$ & $\pm 0.02^{\mathrm{a}}$ & $\pm 0.05^{\mathrm{a}}$ \\
Longitud final (cm) & 9.25 & 8.56 & 8.20 \\
& $\pm 0.37^{\mathrm{a}}$ & $\pm 0.21^{\mathrm{b}}$ & $\pm 1.17^{\mathrm{b}}$ \\
TCE (\% longitud/día) & 0.550 & 0.461 & 0.413 \\
& $\pm 0.038^{\mathrm{a}}$ & $\pm 0.029^{\mathrm{b}}$ & $\pm 0.017^{\mathrm{b}}$ \\
TCA (cm/día) & 0.040 & 0.032 & 0.028 \\
& $\pm 0.004^{\mathrm{a}}$ & $\pm 0.003^{\mathrm{b}}$ & $\pm 0.001^{\mathrm{b}}$ \\
GP (\%) & 64.21 & 51.47 & 45.04 \\
& $\pm 5.66^{\mathrm{a}}$ & $\pm 3.98^{\mathrm{b}}$ & $\pm 2.14^{\mathrm{b}}$ \\
Peso inicial (g) & 2.64 & 2.58 & 2.59 \\
& $\pm 0.09^{\mathrm{a}}$ & $\pm 0.11^{\mathrm{a}}$ & $\pm 0.05^{\mathrm{a}}$ \\
Peso final (g) & 12.90 & 10.32 & 8.90 \\
& $\pm 1.13^{\mathrm{a}}$ & $\pm 0.64^{\mathrm{b}}$ & $\pm 0.42^{\mathrm{b}}$ \\
TCE (\% peso/día) & 1.759 & 1.538 & 1.369 \\
& $\pm 0.133^{\mathrm{a}}$ & $\pm 0.109^{\mathrm{ab}}$ & $\pm 0.042^{\mathrm{b}}$ \\
TCA (g/día) & 0.114 & 0.086 & 0.070 \\
& $\pm 0.013^{\mathrm{a}}$ & $\pm 0.081^{\mathrm{b}}$ & $\pm 0.004^{\mathrm{b}}$ \\
GP (\%) & 389.48 & 300.53 & 243.01 \\
& $\pm 56.52^{\mathrm{a}}$ & $\pm 40.12^{\mathrm{ab}}$ & $\pm 13.00^{\mathrm{b}}$ \\
& 1.193 & 1.736 & 2.213 \\
Rendimiento (kg/m $\left.{ }^{3}\right)$ & $\pm 0.280^{\mathrm{b}}$ & $\pm 0.494^{\mathrm{ab}}$ & $\pm 0.302^{\mathrm{a}}$ \\
& 91.67 & 83.33 & 83.33 \\
Supervivencia (\%) & $\pm 14.63^{\mathrm{a}}$ & $\pm 19.09^{\mathrm{a}}$ & $\pm 14.43^{\mathrm{a}}$ \\
\hline
\end{tabular}

TCE Tasa de crecimiento específico. TCA: Tasa de crecimiento absoluto. GP: Ganancia porcentual. Datos con diferentes letras en superíndice en una misma fila indica diferencia significativa $(p<0.05)$.

La supervivencia de tilapia fue similar (86.11士14.58\%; $F=0.267 ; p=0.775)$ entre tratamientos, pero mayor supervivencia $(91.67 \pm 14.63 \%)$ se obtuvo en 100 alevines/ $\mathrm{m}^{3}$ y las menores supervivencias se obtuvieron a 200 y 300 alevines $/ \mathrm{m}^{3}(83.33 \pm 19.09 \%$ y $83.33 \pm 14.43 \%$, respectivamente). El mayor rendimiento de tilapia $(2.213 \pm 0.302 \mathrm{~kg} /$ $\mathrm{m}^{3}$ ) se obtuvo a 300 alevines $/ \mathrm{m}^{3}$, que fue significativamente mayor $(F=5.661 ; p=0.042)$ al obtenido con 100 alevines $/ \mathrm{m}^{3}(1.193 \pm 0.280$ $\mathrm{kg} / \mathrm{m}^{3}$ ) (Tabla 2). En el último mes, las tilapias cultivadas en alta densidad presentaron pequeñas laceraciones alrededor de la boca.

Calidad del agua. La temperatura del agua de los acuarios se mantuvo en $23.36 \pm 0.12^{\circ} \mathrm{C}$ en promedio. El oxígeno del agua disminuyó conforme aumentó la densidad de tilapia, siendo diferente $(p<0.05)$ la concentración de oxígeno de $6.22 \pm 0.03 \mathrm{mg} / \mathrm{L}$ obtenida a 100 alevines/ $\mathrm{m}^{3}$ con el de $5.75 \pm 0.19 \mathrm{mg} / \mathrm{L}$ a 300 alevines/ $\mathrm{m}^{3}$. El amonio total fue similar $(p>0.05)$ entre tratamientos $(\leq 0.02 \pm 0.00 \mathrm{mg} / \mathrm{L})$. Los nitritos aumentaron $(\mathrm{p}<0.05)$ con la densidad de tilapia, de $0.11 \pm 0.01 \mathrm{mg} / \mathrm{L}$ obtenido a 100 alevines $/ \mathrm{m}^{3}$ hasta $0.18 \pm 0.02 \mathrm{mg} / \mathrm{L}$ a 300 alevines $/ \mathrm{m}^{3}$. En cambio, la dureza total del agua fue diferente $(p<0.05)$ entre tratamientos, siendo mayor en la densidad de 200 alevines $/ \mathrm{m}^{3}(109.33 \pm 1.15$ $\mathrm{mg} / \mathrm{L})$ y menor en 300 alevines $/ \mathrm{m}^{3}(104.00 \pm 0.00$ $\mathrm{mg} / \mathrm{L}$ ) (Tabla 3).

Tabla 3. Parámetros físicos y químicos (media \pm desviación estándar) del agua del cocultivo de $C$. caementarius con $O$. niloticus a diferentes densidades, durante 90 días.

\begin{tabular}{cccc}
\hline \multirow{2}{*}{ Parámetros } & \multicolumn{3}{c}{ Densidad de tilapia (alevines $/ \mathbf{m}^{\mathbf{3}}$ ) } \\
\cline { 2 - 4 } & $\mathbf{1 0 0}$ & $\mathbf{2 0 0}$ & $\mathbf{3 0 0}$ \\
\hline Temperatura $\left({ }^{\circ} \mathrm{C}\right)$ & 23.33 & 23.42 & 23.34 \\
& $\pm 0.11^{\mathrm{a}}$ & $\pm 0.08^{\mathrm{a}}$ & $\pm 0.21^{\mathrm{a}}$ \\
Oxígeno disuelto & 6.22 & 6.06 & 5.75 \\
(mg/L) & $\pm 0.03^{\mathrm{a}}$ & $\pm 0.28^{\mathrm{ab}}$ & $\pm 0.19^{\mathrm{b}}$ \\
& 0.00 & 0.02 & 0.02 \\
Amonio total (mg/L) & $\pm 0.00^{\mathrm{a}}$ & $\pm 0.00^{\mathrm{a}}$ & $\pm 0.00^{\mathrm{a}}$ \\
& 0.11 & 0.18 & 0.18 \\
Nitritos (mg/L) & $\pm 0.01^{\mathrm{a}}$ & $\pm 0.03^{\mathrm{b}}$ & $\pm 0.02^{\mathrm{b}}$ \\
Dureza total (mg & 107.33 & 109.33 & 104.00 \\
$\left.\mathrm{CaCO}_{3} / \mathrm{L}\right)$ & $\pm 1.15^{\mathrm{a}}$ & $\pm 1.15^{\mathrm{b}}$ & $\pm 0.00^{\mathrm{c}}$ \\
\hline
\end{tabular}

Datos con diferentes letras en superíndice en una misma fila indica diferencia significativa $(p<0.05)$.

\section{DISCUSIÓN}

La temperatura del agua $\left(23.36^{\circ} \mathrm{C}\right)$ estuvo dentro de lo registrado para el ambiente natural del camarón (13) pero no fue apropiada para tilapia, pues el mayor crecimiento y utilización de alimento en tilapia se logra entre 27 a $32^{\circ} \mathrm{C}$ (21) lo que debe haber contribuido con el retraso del crecimiento. La disminución de la concentración de oxígeno del agua (6.22 a $5.75 \mathrm{mg} / \mathrm{L}$ ) conforme incrementó la densidad de 
tilapia fue significativa, pero estuvo dentro de lo informado para la especie (20), así como en otros cocultivos similares con tilapia y camarón $(22,23)$. El amonio total $(\leq 0.02 \mathrm{mg} / \mathrm{L})$ similar entre tratamientos y los nitritos ( $\leq 0.18 \mathrm{mg} / \mathrm{L}$ ) que aumentaron significativamente con la densidad, no fueron perjudiciales, pues estuvieron dentro de lo informado para el ambiente natural de las especies $(20,21,24)$. Estos resultados indican que el sistema de cultivo empleado mantuvo los niveles de productos nitrogenados del agua aceptables para las especies, a pesar del incremento de la biomasa y principalmente el de tilapia en alta densidad, que es la que ocasiona deterioro de la calidad del agua (9).

La longitud final promedio $(6.46 \mathrm{~cm})$ y el peso final promedio $(9.37 \mathrm{~g})$, así como los parámetros de crecimiento en longitud y peso del camarón fueron similares entre tratamientos (Tabla 1). Sin embargo, las mayores ganancias porcentuales en longitud se obtuvieron en el cocultivo con tilapia a 100 y 200 alevines/m ${ }^{3}$ (10.29\% y $10.45 \%$, respectivamente); similar tendencia se obtuvo con la tasa de crecimiento específica y la tasa de crecimiento absoluta en longitud. Estos resultados están de acuerdo con los obtenidos en la misma especie de camarón cultivada en recipientes individuales, pero de trabajos que evaluaron otros estímulos $(25,26)$.

Las mayores ganancias porcentuales en peso del camarón fueron obtenidas en el cocultivo con tilapia a 200 y 300 alevines $/ \mathrm{m}^{3}$ (25.41\% y $23.29 \%$, respectivamente), pero menores a los obtenidos previamente en la misma especie de camarón $(15,25,27)$. Estos resultados fueron debido a que algunos ejemplares machos no pudieron culminar con el proceso de la ecdisis y realizaron autotomía de quelípodos para sobrevivir, con lo cual perdieron peso. La pérdida de un quelípodo en esta especie de camarón macho reduce el peso entre 12 y $22 \%$, y la pérdida de ambos quelípodos entre el 30 y $40 \%$ $(15,28)$. En Pagurus middendorffii, hay supresión del crecimiento somático por la regeneración del quelípodo mayor (29).

La supervivencia de los camarones machos de C. caementarius fue alta ( 94.44 y $88.89 \%$ ) y sin diferencia entre tratamientos. En $C$. quadricarinatus cultivado en recipientes individuales la supervivencia fue entre 73 a 98\% (16) y de 64 a $67 \%$ en Scylla paramamosain (30) y hasta $86 \%$ en $H$. gammarus (31). En la investigación, la disminución de la supervivencia de los camarones cultivados en recipientes individuales no se debió a la presencia de tilapia ni a la densidad de esta en el sistema de cocultivo, sino al síndrome de la ecdisis incompleta que ocasionó muertes porque la exuvia quedó atrapada en los periópodos. Este síndrome sucede en $C$. caementarius debido a deficiencias nutricionales $(25,26)$, mas no al tamaño de los recipientes individuales empleados (15). En estudios previos con la especie cultivada en los mismos recipientes, se obtuvo supervivencias $>61 \%$, donde las muertes se debieron a ecdisis incompleta $(15,25)$. Supervivencia $>90 \%$ se obtuvo en C. quadricarinatus dentro de los recipientes individuales, aunque no se informaron las causas (16). Sin embargo, no se conoce si el síndrome de muerte por muda ocurre en el ambiente natural. En la presente investigación se corrobora la eficiencia del sistema de cultivo individual para mantener alta supervivencia del camarón al evitar la interacción intraespecífica, y se demuestra por primera vez que el sistema disminuye la interacción con tilapia. Este sistema permitirá realizar cocultivo con otras especies de peces e incluso con otros crustáceos de agua dulce.

La muerte por ecdisis incompleta, así como la pérdida de quelípodos durante la ecdisis, no solo afectó la supervivencia y el crecimiento sino también el rendimiento productivo de $C$. caementarius $\left(0.263\right.$ a $\left.0.308 \mathrm{~kg} / \mathrm{m}^{2}\right)$, aunque estuvo dentro de lo obtenido previamente en la misma especie cultivada en los mismos recipientes $\left(0.241\right.$ y $\left.1.049 \mathrm{~kg} / \mathrm{m}^{2}\right)(15,26)$. Es conveniente continuar con las investigaciones para evitar que los camarones en cultivo pierdan los quelípodos, toda vez que $C$. caementarius se comercializa entero y la pérdida de quelípodos reduce significativamente el peso del camarón (28) y por tanto el rendimiento productivo, como ha sido demostrado en la investigación.

El crecimiento de $O$. niloticus fue afectado inversamente por el incremento de la densidad durante el periodo de cultivo, donde los parámetros de crecimiento fueron mayores cuando se cultivaron a 100 alevines $/ \mathrm{m}^{3}$, a excepción de la tasa de crecimiento específica y la ganancia porcentual en peso que fueron similares hasta con 200 alevines/m³. Estos resultados están de acuerdo con lo informado en tilapia, donde mayor crecimiento en peso se obtuvo con 100 peces $/ \mathrm{m}^{3}$ (8) y, densidades mayores de 150 alevines $/ \mathrm{m}^{3}$ ocasionan competencia por alimento y espacio que afectan el crecimiento (9). En cambio, la disminución significativa del crecimiento de tilapia en alta densidad (300 alevines $/ \mathrm{m}^{3}$ ) podría ser consecuencia de la interacción intraespecífica, toda vez que se 
conoce que en alta densidad hay cambios en el metabolismo de la tilapia por incremento de energía para contrarrestar el estrés (9). Además, el empleo de alimento no formulado para la especie que, aunque tuvo $30 \%$ de proteína cruda y $8.1 \%$ de lípidos totales, es probable que algunos otros nutrientes faltaron o fueron deficientes que no contribuyeron con la nutrición. En la misma especie de tilapia alimentada con una dieta con $30 \%$ de proteína, se produce mayor retención de proteínas y aminoácidos, los cuales mejoran el crecimiento (32). Así mismo, los ácidos grasos como el ácido linoleico, el eicosapentanoico y docosahexanoico, son beneficiosos para el crecimiento y el estado de salud (33).

En el cocultivo de camarón/tilapia, es necesario emplear una dieta específica para cada especie, pues las tilapias del presente estudio consumieron solo alimento formulado para camarón, pero en baja proporción. El nivel de alimentación empleado ( $5 \%$ ) para alevines de tilapia, que se pensó que incrementaría con el alimento que sale de los recipientes de cultivo de camarones, no fue suficiente en cantidad ni en calidad. En alevines de tilapia se emplea como ración diaria el $10 \%$ de la biomasa (34). Esta deficiencia de alimento para tilapia fue más evidente en el último mes de cultivo, al observarse pequeñas laceraciones en la boca de los peces que indicaría agresividad por competencia por alimento. Este comportamiento debe haber ocasionado alguna infección que ocasionó muerte de los peces y disminución de la supervivencia (83\%) en alta densidad, aunque fue similar ( $91 \%)$ en baja densidad. La tilapia es territorialista y el incremento de la densidad afecta el crecimiento debido a la competencia por alimento (35). Es conveniente continuar con las investigaciones que permitan mejorar el manejo del cocultivo de camarones con peces en lo relacionado con la nutrición y con las condiciones ambientales requeridos por las especies, para lograr el aprovechamiento integral del cuerpo de agua sin afectar el ambiente.

El rendimiento productivo de tilapia fue significativamente mayor con el incremento de la densidad, aun cuando el crecimiento fue menor. Estos resultados podrían ser consecuencia del tamaño inicial de los peces, pues nosotros empleamos alevines de $2.61 \mathrm{~g}$ con el cual se logró incrementar el rendimiento desde 1.193 hasta $2.213 \mathrm{~kg} / \mathrm{m}^{2}$. El cultivo con pequeños alevines de tilapia $(0.80 \mathrm{~g})$ en policultivo con $M$. rosenbergii se logra rendimientos entre 0.162 a $0.254 \mathrm{~kg} / \mathrm{m}^{2}$ (36). En cambio, cuando se emplean grandes juveniles $(29.54 \mathrm{~g})$, cultivados en jaulas, se logra rendimientos de 13.9 a 28.56 $\mathrm{kg} / \mathrm{m}^{3}$ que incrementa con la densidad (37).

En la investigación, se demostró una vez más que los machos de $C$. caementarius soportan el cultivo en espacio físico reducido como los recipientes individuales empleados en esta y otras investigaciones $(15,25,26,27)$. Sin embargo, la interacción agresiva del camarón hacia la tilapia fue evidente por que los camarones intentaron capturar al pez cuando sacaron sus quelípodos por los orificios del recipiente de cultivo, pero no hubo muertes de tilapia. Estos resultados, corroboran el carácter agresivo del camarón macho (15). Además, el comportamiento agresivo del camarón evidencia que, en policultivo tradicional, ambas especies se verían afectadas por incremento del canibalismo y por interacción física, como sucede en otras especies. En el policultivo de $O$. niloticus con $P$. acanthophorus, hay competencia por espacio, alimento e incluso depredación por la tilapia durante la muda del crustáceo (38). Así mismo, en el policultivo de $M$. rosenbergii con $O$. niloticus la competencia por alimento y espacio afectan la producción (5). Es conveniente investigar el comportamiento de las especies en cocultivo, pero a nivel de estanques seminaturales, así como la viabilidad técnica y económica, como ha sido establecido para otras combinaciones de organismos acuáticos en cocultivo en estanques (39), tendiente a establecer una producción comercial.

En conclusión, el crecimiento y la supervivencia del camarón $C$. caementarius fue afectado por el síndrome de muerte por muda, mas no por la presencia de la densidad de tilapia en el sistema de cocultivo, pero no hubo diferencias significativas entre tratamientos. En cambio, mayor crecimiento de tilapia $O$. niloticus se obtuvo con 100 alevines $/ \mathrm{m}^{3}$, y la supervivencia fue similar entre tratamientos; pero mayor rendimiento se logró con 300 alevines $/ \mathrm{m}^{3}$. El uso de alimento balanceado específico para ambas especies es necesario para lograr mayor crecimiento. Además, se requiere de un estudio económico que permita determinar la viabilidad del cocultivo comercial camarón/tilapia, pero en estanques seminaturales.

\section{Conflicto de interés}

Los autores no tienen ningún conflicto de intereses. 


\section{REFERENCIAS}

1. Fitzsimmons KM, Shahkar E. Tilapia-Shrimp polyculture. In: Perschbacher PW, Stickney RR (Eds). Tilapia in intensive co-culture. Wiley Blackwell: EU; 2017. https://www. doi.org/10.1002/9781118970652.ch10 .

2. New MB, Valenti WC. Tilapia-Macrobrachium polyculture. In: Perschbacher PW, Stickney RR. (Eds) Tilapia in intensive co-culture. Wiley Blackwell: EU; 2017. https://www. doi.org/10.1002/9781118970652.ch11 _

3. Schmalenbach I, Buchholz F, Franke HD, Saborowski R. Improvement of rearing conditions for juvenile lobster (Homarus gammarus) by co-culturing with juvenile isopods (Idotea emarginata). Aquac. 2009; 289(3-4):297-303. https://www.doi. org/10.1016/j.aquaculture.2009.01.017 _

4. Rohmana D, Surawidjaja EH, Sukenda S, Ekasari J. Water quality and production performance of catfish-prawn co-culture with organic carbon source addition. Aquacult Int. 2015; 23:267-276. https:// www.doi.org/10.1007/s10499-014-9814-2

5. Tidwell JH, Coyle SD, Bright LA. Polyculture of Nile tilapia, Oreochromis niloticus, either confined in cages or unconfined in freshwater prawn, Macrobrachium rosenbergii, ponds. J World Aquac Soc. 2010; 41(4):616-625. https://www.doi.org/10.1111/j.17497345.2010.00402.x

6. Tesfahun A, Temesgen M. Food and feeding habits of Nile tilapia Oreochromis niloticus (L.) in Ethiopian water bodies: A review. Int J Fish Aquat Stud. 2018; 6(1):43-47. http:// www.fisheriesjournal.com/archives/2018/ vol6issue1/PartA/5-6-54-506.pdf

7. Nunoo FKE, Asase A. Comparative growth performance of Oreochromis niloticus (Linnaeus, 1758) in cages at different stocking densities. Int J Fish Aquat Stud. 2017; 5(4):279-283. http://www. fisheriesjournal.com/archives/2017/ vol5issue4/PartD/5-4-4-982.pdf
8. Araujo G, Rodrigues J, Da Silva J, Farias W. Cultivo da tilápia do Nilo Oreochromis niloticus em tanques-rede circulares em diferentes densidades de estocagem. Biosci J. 2010; 26(3):428-434. http://www.seer. ufu.br/index.php/biosciencejournal/article/ view/7080

9. Abdel-Tawwab M. Effects of dietary protein levels and rearing density on growth performance and stress response of Nile tilapia, Oreochromis niloticus (L.). Int Aquat Res. 2012; 4(3):1-12. https://www.doi. org/10.1186/2008-6970-4-3

10. Yakubu A, Obi A, Okonji V, Ajiboye O, Adams T, Olaji $E$, et al. Growth performance of Nile tilapia (Oreochromis niloticus) as affected by stocking density and feed types in water flow through system. World J Fish Marine Sci. 2012; 4(3):320-324. http://www.doi. org/10.5829/idosi.wjfms.2012.04.03.6230

11. Shubha M, Reddy S. Effect of stocking density on growth, maturity, fecundity, reproductive behaviour and fry production in the mouth brooding cichlid Oreochromis mossambicus (Peters). Afr J Biotechnol. 2011; 10(48):9922-9930. http://dx.doi. org/10.5897/AJB11.697

12. Moscoso V. Catálogo de crustáceos decápodos y estomatópodos del Perú. Bol Inst Mar Perú. 2012; 27(1-2):8-207. http://biblioimarpe. imarpe.gob.pe/handle/123456789/2190

13. Wasiw J, Yépez V. Evaluación poblacional del camarón Cryphiops caementarius en ríos de la costa sur del Perú. Rev Inv Vet Perú. 2015; 26(2):166-181. http://dx.doi. org/10.15381/rivep.v26i2.11103

14. Ministerio de la Producción. Anuario estadístico pesquero y acuícola 2017. La actividad productiva del sector en números, Perú. 2018. http://ogeiee.produce.gob.pe/ images/Anuario/Pesca_2017.pdf 
15. Reyes W. Effect of culture container on the survival and growth of male Cryphiops caementarius in individualized systems. Revista Bio Ciencias. 2016; 3(4):311325. https://www.doi.org/10.15741/ revbio.03.04.06

16. Manor R, Segev R, Pimenta M, Aflalo ED, Sagi A. Intensification of redclaw crayfish Cherax quadricarinatus culture II. Growout in a separate cell system. Aquacult Eng. 2002; 26(4):263-276. https://www.doi. org/10.1016/S0144-8609(02)00035-3

17. Drengstig A, Bergheim A. Commercial land-based farming of European lobster (Homarus gammarus L.) in recirculating aquaculture system (RAS) using a single cage approach. Aquacult Eng. 2013; 53:14-18. https://www.doi.org/10.1016/j. aquaeng.2012.11.007

18. Méndez M. Claves de identificación y distribución de los langostinos y camarones (Crustacea: Decapoda) del mar y ríos de la costa del Perú. Bol Inst Mar Perú. 1981; 5:1-169. http://biblioimarpe.imarpe.gob. pe/handle/123456789/1028

19. Reyes W, Terrones S, Baltodano I. Effects chelipeds regeneration in molting and growth of male Cryphiops caementarius Molina 1782 (Decapod, Palaemonidae). Revista Bio Ciencias. 2017; 4(4): 1-18. http://dx.doi. org/10.15741/revbio.04.04.05

20. Cornejo J, Pérez L, Reyes W. Effect of Saccharomyces cerevisiae yeast in the diet of male shrimp Cryphiops caementarius (Crustacea, Palaemonidae) on total and differential hemocytes count. Revista Bio Ciencias. 2015; 3(3):173-186. http:// dx.doi.org/10.15741/revbio.03.03.04

21. Pandit NP, Nakamura M. Effect of high temperature on survival, growth and feed conversion ratio of Nile tilapia, Oreochromis niloticus. Our Nature. 2012; 8(1):3219-224. https://doi.org/10.3126/on.v8i1.4331 _

22. López-Gómez C, Ponce-Palafox T, CastilloVargasmachuca S, Puga-López D, CastilloCampo LF, García-Ulloa M. Evaluation of two mix-cultures of white shrimp (Litopenaeus vannamei) with red tilapia hybrid and spotted rose snapper (Lutjanus guttatus) in intensive indoor brackish water tanks. Lat Am J Aquat Res. 2017; 45(5): 922-929. http://www.doi.org/10.3856/vol45-issue5fulltext-7
23. Juárez-Rosales J, Ponce-Palafox T, RománGutiérrez AD, Otazo-Sánchez OM, PulidoFlores G, Castillo-Vargasmachuca SG. Effects of White shrimp (Litopenaeus vannamei) and tilapia nilotica (Oreochromis niloticus var, Spring) in monoculture and coculture systems on water quality variables and production in brackish low-salinity water earthen ponds during rainy and dry seasons. Span J Agric Res. 2019; 17(3): e0605. https://www.doi.org/10.5424/ sjar/2019173-14938

24. Wasiw J, Yépez V. Evolución de la condición poblacional del camarón Cryphiops caementarius en el río Cañete (2000-2015). Rev Inv Vet Perú. 2017; 28(1):13-32. http:// dx.doi.org/10.15381/rivep.v28i1.12942

25. Acosta A, Quiñones D, Reyes W. Efecto de dietas con lecitina de soya en el crecimiento, muda y supervivencia de machos del camarón de río Cryphiops caementarius (Crustacea: Palaemonidae). Sci Agropecu. 2018; 9(1):143-151. http://www.doi. org/10.17268/sci.agropecu.2018.01.15

26. Terrones S, Reyes W. Efecto de dietas con ensilado biológico de residuos de molusco en el crecimiento del camarón Cryphiops caementarius y tilapia Oreochromis niloticus en cocultivo intensivo. Sci Agropecu. 2018; 9(2):167-176. http://www.doi. org/10.17268/sci.agropecu.2018.02.01

27. Ramírez M, Cántaro R, Reyes W. Growth and survival of males of Cryphiops caementarius (Palaemonidae) with diets supplemented with common salt. Lat Am J Aquat Res. 2018; 46(2):469-474. http://www.doi. org/10.3856/vol46-issue2-fulltext-22

28. Reyes W. El síndrome de la ecdisis incompleta en machos adultos de Cryphiops caementarius (Crustacea: Palaemonidae) y sus consecuencias en cultivo intensivo. Rev Inv Vet Perú. 2018; 29(1):68-374. http:// dx.doi.org/10.15381/rivep.v29i1.14200

29. Yasuda CI, Matsuo K, Wada S. Rapid regeneration of the major cheliped in relation to its function in male-male contests in the hermit crab Pagurus middendorffii. Plankton Benthos Res. 2014; 9(2):122-131. https://www.doi.org/10.3800/pbr.9.122 
30. Islam ML, Siddiky MNSM, Yahya K. Growth, survival and intactness of green mud crab (Scylla paramamosain) broodstock under different captive grow out protocols. SAARC J Agri. 2018; 16(1):169-180. http://dx.doi. org/10.3329/sja.v16i1.37432

31. Daniels $C L$, Wills $B$, Ruiz-Perez $M$, Miles E, Wilson RW, Boothroyd D. Development of sea-based container culture for rearing European lobster (Homarus gammarus) around South West England. Aquac. 2015; 448:186-195. http://dx.doi.org/10.1016/j. aquaculture.2015.05.026

32. Teodósico R, Engrola $S$, Colen $R$, Masagounder K, Aragão C. Optimizing diets to decrease environmental impact of Nile tilapia (Oreochromis niloticus) production. Aquacult Nutr. 2019; 26(2): 422-431. http://www.doi.org/10.1111/anu.13004

33. Liu Y, Wen JJ, Ning LJ, Jiao JG, Qiao F, Chen LQ, et al. Comparison of effects of dietary-specific fatty acids on growth and lipid metabolism in Nile tilapia. Aquacult Nutr. 2019; 25(4):862-872. https://doi. org/10.1111/anu.12906

34. Bessa AP, Borges CMS, Silva F, Henry-Silva GG. Polyculture of Nile tilapia and shrimp at different stocking densities. R Bras Zootec. 2012; 41(7):1561-1569. http://dx.doi. org/10.1590/S1516-35982012000700002

35. Costa ÂAP, Roubach R, Dallago BSL, Bueno GW, McManus C, Bernal FEM. Influence of stocking density on growth performance and welfare of juvenile tilapia (Oreochromis niloticus) in cages. Arq Bras Med Vet Zootec. 2017; 69(1):243-251. http:// dx.doi.org/10.1590/1678-4162-8939
36. Tidwell JH, Coyle SD, Bright LA. Polyculture of Nile tilapia, Oreochromis niloticus, either confined in cages or unconfined in freshwater prawn, Macrobrachium rosenbergii, ponds. J World Aquac Soc. 2010; 41(4):616-625. https://www.doi.org/10.1111/j.17497345.2010.00402.x

37. Osofero S, Otubusin S, Daramola J. Effect of stocking density on tilapia (Oreochromis niloticus Linnaeus 1757) growth and survival in bamboo-net cages trial. Afr J Biotechnol. 2009; 8(7):1322-1325. https:// www.ajol.info/index.php/ajb/article/ view/60113/48369

38. Hernández-Vergara MP, Cruz-Ordóñez SB, Pérez-Rostro CI, Pérez-Legaspi A. Polyculture of crayfish (Procambarus acanthophorus) and Nile tilapia (Oreochromis niloticus) as a strategy for sustainable water use. Hidrobiológica. 2018; 28(1):11-15. https:// www.doi.org/10.24275/uam/izt/dcbs/ hidro/2018v28n1/HernandezV

39. Mondal A, Bhattacharya S, Mitra A, Sundaray JK, Mohanty RK. Performance evaluation of mud crab Scylla olivacea (Herbst, 1896) coculture with different fish species in confined brackishwater ponds. Aquac. 2020; 522:735125. https://doi.org/10.1016/j. aquaculture.2020.735125 